\title{
Pros and cons of permissive hypercapnia in patients with subarachnoid haemorrhage and ARDS
}

\author{
Marcus H. T. Reinges
}

Received: 27 July 2010 / Accepted: 29 July 2010 / Published online: 17 August 2010

(C) Springer-Verlag 2010

Petridis and colleagues describe their results of intracranial pressure (ICP) analysis in 12 patients after subarachnoid haemorrhage $(\mathrm{SAH})$ treated with permissive hypercapnia due to acute respiratory distress syndrome (ARDS) [6].

The study is of interest for physicians practicing neurosurgical intensive care, since many patients who suffer from severe SAH develop serious pulmonary problems during their stay in the intensive care unit. In general, these patients have a very high mortality rate and, therefore, the authors have to be congratulated on their good results.

However, there are several aspects of the paper which should compel the reader to interpret the results very carefully:

On the one hand, the regimen described is in contrast to the widely accepted opinion that hypercapnia should be avoided in brain-damaged patients, since hypercarbia potentially leads via acidosis-induced cerebral vasodilatation to an increase of intracranial blood volume which again leads to an increase of ICP $[1-3,5,7]$. Therefore, patients with elevated ICP have notably been excluded from studies on permissive hypercapnia [1]. Also other side effects of hypercarbia-especially severe acidosis with its deleterious effects in intensive care medicinehave to be kept in mind.

On the other hand, there are animal studies demonstrating a beneficial effect of hypercapnia on the central nervous system: Zhou and colleagues concluded in their recently published paper that mild to moderate hypercap-

\section{H. T. Reinges $(\bowtie)$}

Neurosurgery, RWTH Aachen University,

Pauwelstr. 30,

52057 Aachen, Germany

e-mail: mreinges@ukaachen.de nia is neuroprotective after transient global cerebral ischemia-reperfusion injury, whereas severe hypercapnia increased brain injury [8]. Hare and colleagues hypothesized in their paper that the combined effect of increased cerebral blood flow, $\mathrm{PaO} 2$, and reduced $\mathrm{pH}$ collectively contribute to augmenting cerebral tissue oxygen tension during hypercapnia [4].

Thus, at present a generally accepted recommendation about permissive hypercapnia in brain injured patients cannot be given. In my opinion, permissive hypercapnia should to date only be administered in patients with a therapy refractory ARDS in whom advanced methods to reduce hypercarbia - e.g., jet ventilation or interventional lung assist devices - if available - have not been successful, i.e., as a form of an individual treatment attempt. A continuously performed ICP monitoring seems to be mandatory - as performed by the authors in the paper [6].

Furthermore, it has to be pointed out that there are some methodological weaknesses of the paper [6]: This is not a controlled randomized clinical study, but the report of a retrospective observation of non-consecutive patients. Another important point is that ICP measurements have been performed while the external ventricular drainage (EVD) was still open: Thus, it is unclear whether hypercapniainduced intracranial vasodilatation has not been compensated by an increase of CSF outflow via the EVD.

As a conclusion, the treatment reported in the paper by Petridis and colleagues [6]-even if there are really interesting aspects, especially the potentially neuroprotective role of mild to moderate hypercapnia [8] - cannot generally be recommended until studies with a better design, especially controlled, randomized studies, have confirmed the results.

Conflicts of interest None. 


\section{References}

1. Acute Respiratory Distress Syndrome Network (2000) Ventilation with lower tidal volumes as compared with traditional tidal volumes for acute lung injury and the acute respiratory distress syndrome. N Engl J Med 342:1301-1308

2. Amato MB, Barbas CS, Medeiros DM, Magaldi RB, Schettino GP, Lorenzi-Filho G, Kairalla RA, Deheinzelin D, Munoz C, Oliveira R, Takagaki TY, Carvalho CR (1998) Effect of a protectiveventilation strategy on mortality in the acute respiratory distress syndrome. N Engl J Med 338:347-354

3. Bigatello LM, Patroniti N, Sangalli F (2001) Permissive hypercapnia. Curr Opin Crit Care 7:34-40

4. Hare GM, Kavanagh BP, Mazer CD, Hum KM, Kim SY, Coackley C, Barr A, Baker AJ (2003) Hypercapnia increases cerebral tissue oxygen tension in anesthetized rats. Can J Anaesth 50:1061-1068
5. Nakahata K, Kinoshita H, Hirano Y, Kimoto Y, Iranami H, Hatano Y (2003) Mild hypercapnia induces vasodilation via adenosine triphosphate-sensitive $\mathrm{K}+$ channels in parenchymal microvessels of the rat cerebral cortex. Anesthesiology 99:1333-1339

6. Petridis AK, Doukas A, Kienke S, Maslehaty H, Mahvash M, Barth $\mathrm{H}$, Mehdorn HM (2010) The effect of lung-protective permissive hypercapnia in intracerebral pressure in patients with subarachnoid haemorrhage and ARDS. A retrospective study. Acta Neurochim. doi:10.1007/s00701-010-0761-z

7. van Hulst RA, Hasan D, Lachmann B (2002) Intracranial pressure, brain $\mathrm{PCO} 2, \mathrm{PO}$, and $\mathrm{pH}$ during hypo- and hyperventilation at constant mean airway pressure in pigs. Intensive Care Med 28:6873

8. Zhou Q, Cao B, Niu L, Cui X, Yu H, Liu J, Li H, Li W (2010) Effects of permissive hypercapnia on transient global cerebral ischemia-reperfusion injury in rats. Anesthesiology 112:288297 